\title{
Molecular Organization in a Nematogen: PBPCN - A Computational Analysis Based on Quantum Mechanics
}

\author{
Durga Prasad Ojha, Devesh Kumar, and V. G. K. M. Pisipati \\ Centre for Liquid Crystal Research and Education (CLCRE), Faculty of Physical Sciences, \\ Nagarjuna University, Nagarjuna Nagar (A.P.) - 522 510, India \\ Reprint requests to Prof. V. G. K. M. P.; E-mail: venkata_pisipati@ hotmail.com
}

Z. Naturforsch. 56 a, 730-734 (2001); received September 7, 2001

\begin{abstract}
A computational analysis has been carried out to determine the configurational preference of a pair of 4'- $n$-pentyloxy-4-biphenylcarbonitrile (PBPCN) molecules with respect to translatory and orientational motions. The $\mathbf{C N D O} / \mathbf{2}$ method has been employed to evaluate the net atomic charge and atomic dipole components at each atomic centre of the molecule. Modified RayleighSchrödinger perturbation theory along with multicentered-multipole expansion method has been employed to evaluate long-range intermolecular interactions, while a ' 6 -exp' potential function has been assumed for short-range interactions. On the basis of stacking, in-plane and terminal interaction energy calculations, all possible geometrical arrangements of molecular pair have been considered. It has been observed that the molecule has a strong preference for stacking through a particular face, while the other configurations, such as stacking through the other face, in-plane and terminal interactions show, in general, an aligned structure along molecular axis. The results are discussed in the light of experimental as well as other theoretical observations.
\end{abstract}

Key words: PBPCN; Nematogen; Interaction Energy; Computer Simulation.

\section{Introduction}

Liquid crystalline phases have always partial orientational order of the molecules, and some have also partial positional order of the molecules. Since this occurs frequently in natural and synthetic materials, liquid crystals are of considerable fundamental and applied interest [ $1-3]$. The peculiar changes that ooccur at phase transitions are primarily governed by intermolecular interactions between sides, planes and ends of a pair of molecules [4]. The molecular interactions of mesogenic compounds have been calculated by several workers [5 - 7] basing on the Buckingham potential with modified Rayleigh-Schrödinger perturbation theory. Attempts were also made to correlate the mesogenic properties with molecular interactions.

It has been observed that the pair potential between such molecules is anisotropic and that the minimum energy configuration corresponds to the crystallographic structure of the molecule in the solid state.

Recently $[8,9]$ the results of theoretical investigations on the role of intermolecular interactions in mesogenic compounds have been reported. One of the limitations of the work was that interaction energy calculations were carried out only through one face.

In the present communication, an attempt has been made to interpret the results of interaction energy calculations through both faces, sides and ends, of a molecular pair of PBPCN molecules at an intermediate distance of $\pm 7 \AA$ for stacking and $\pm 7 \AA$ for in-plane interactions. Similarly, a distance of $\pm 20 \AA$ has been kept for terminal interactions.

The thermodynamic parameters reveal that PBPCN shows a nematic phase at $47.5^{\circ} \mathrm{C}$ and exhibits an isotropic melt at $67.5^{\circ} \mathrm{C}$ [10].

\section{Computational Details}

The CNDO/2 method [11] has been employed to compute the net atomic charge and dipole moment at each atomic centre of the molecule. The molecular geometry of PBPCN has been constructed on the basis of the published crystallographic data with standard values of bond lengths and bond angles [10]. The length of the most extended conformation was found to be $17.5 \AA$, which is the calculated length of the 
molecule in the crystalline state. The apparent length of the molecule in the nematic phase was found to be $24.5 \AA$, which is nearly 1.4 times the calculated length in crystalline state [10].

According to the second order perturbation theory, as modified for intermediate range interactions [12], the total interaction energy $\left(E_{\text {total }}\right)$ between a pair molecules is given by

$$
E_{\text {total }}=E_{\mathrm{el}}+E_{\mathrm{pol}}+E_{\mathrm{disp}}+E_{\mathrm{rep}},
$$

where $E_{\mathrm{el}}, E_{\mathrm{pol}}, E_{\mathrm{disp}}$, and $E_{\text {rep }}$, respectively, represent the electrostatic, polarization, dispersion and repulsion components.

The electrostatic term is expressed as

$$
E_{\mathrm{el}}=E_{\mathrm{QQ}}+E_{\mathrm{QMI}}+E_{\mathrm{MIMI}}+\ldots,
$$

where $E_{\mathrm{QQ}}, E_{\mathrm{QMI}}$, and $E_{\mathrm{MIMI}}$ etc. are the monopole-monopole, monopole-dipole and dipole-dipole etc. terms, respectively. In fact, the inclusion of higher order multipoles does not affect significantly the electrostatic interaction energy, calculations upto the dipole-dipole term giving satisfactory results [13], as has been done here. The necessary formulae may be found in [9].

The origin on an atom has been chosen close to the centre of mass of the molecule, the $X$-axis along the long molecular axis, the $Y$-axis in the plane of the molecule and the $Z$-axis perpendicular to the molecular plane.

\section{Results and Discussion}

The molecular geometry of PBPCN is shown in Figure 1. The total energy, binding energy, total dipole moment and its components are listed in Table 1. The results of the interaction energy calculations are discussed below.

\section{Stacking Interactions}

Of the molecular pair, one molecule is fixed in the $X-Y$ plane such that the $X$-axis lies along the long molecular axis, while the other molecule is kept at a separation of $7 \AA$ along the $Z$-axis with respect to the fixed molecule. Computations have been carried out on both faces $F_{1}$ and $F_{2}$, i. e. $+7 \AA$ and $-7 \AA$ interplanar separation between the two molecules. The choice of the distance has been made to eliminate the possibility of van der Waals contacts completely and to keep the molecule within short and medium range interaction.
Table 1. Total energy ${ }^{\mathrm{a}}$, binding energy ${ }^{\mathrm{b}}$, total dipole moment and its components of the PBPCN molecule. Total energy = -172.11 atomic unit, Binding energy $=-19.85$ atomic unit, Total dipole moment $=2.71$ debyes

\begin{tabular}{lccr}
\hline & \multicolumn{3}{c}{ Components } \\
& $X$ & $Y$ & $Z$ \\
\hline Densities $^{+}$ & 0.536 & 1.315 & -0.020 \\
sp* $^{*}$ & -0.474 & 1.391 & -0.018 \\
pd** $^{* *}$ & 0.000 & 0.000 & 0.000 \\
Total $^{++}$ & 0.062 & 2.705 & -0.038 \\
\hline
\end{tabular}

a The total energy corresponds to the sum of the atomic and electronic energies of all the constituents of the molecule in the equilibrium geometry. ${ }^{\mathrm{b}}$ The binding energy of a molecule is the difference between the energy of the equilibrium molecular geometry and the sum of the atomic energies of the constituent atoms. ${ }^{+}$Contribution to dipole moment components due to electron densities. ${ }^{*}$ sp-hybridization moment; ${ }^{* *}$ pd-hybridization moment. ${ }^{++}$Sum of electron density and hybridization contribution to the dipole moment component.

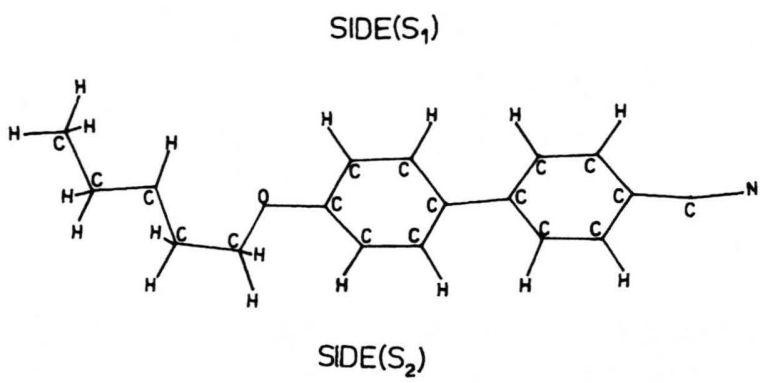

Fig. 1. $X-Y$ projection of the molecular geometry of PBPCN with various atoms.

Rotations about the $Z$-axis have been given at intervals of $10^{\circ}$, and the interaction energy at each point has been calculated. The minimum energy so obtained is then taken as starting point, and the entire process is repeated at smaller intervals. The association energy has been minimized with respect to translation and rotation about the two axes. An accuracy of $0.1 \AA$ in translation and $1^{\circ}$ in rotation of one molecule with respect to other has been achieved. The final lowest stacked geometry was thus obtained through face $\mathrm{F}_{1}$ at an interplanar separation of $3.4 \AA$ with energy $-12.73 \mathrm{kcal} / \mathrm{mole}$ and face $\mathrm{F}_{2}$ at an interplanar separation of $3.6 \AA$ with energy $-12.83 \mathrm{kcal} / \mathrm{mole}$. These configurations agree with those obtained from crystallographic studies [10].

The variation of the intermolecular interaction energy components through face $F_{1}$ with respect to rotation about the $X$-axis is shown in Figure 2. The dominating role of the dispersion energy is obvious, 


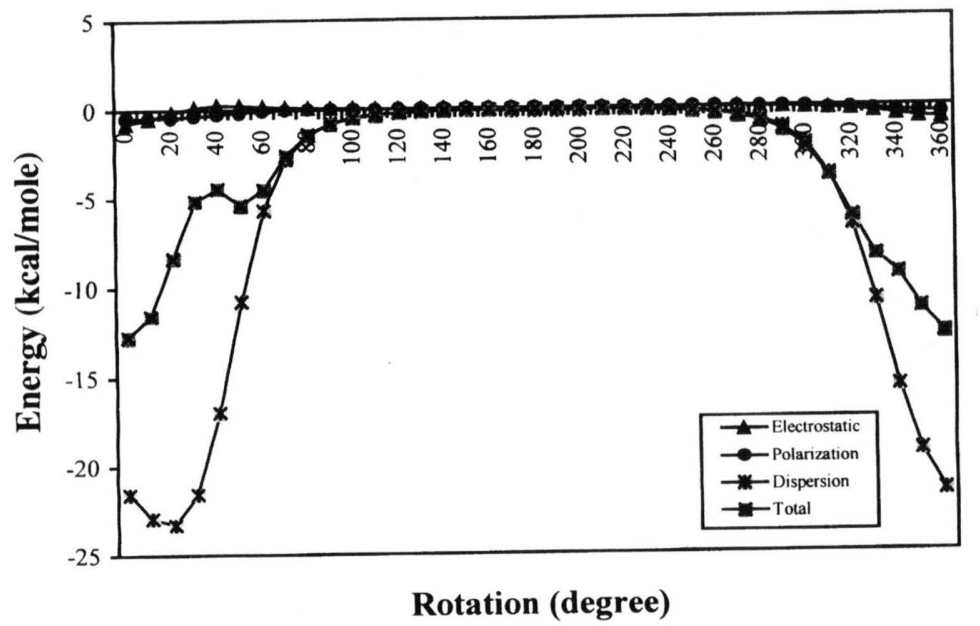

Fig. 2. Variation of the interaction energy components with respect to rotation about the $X$-axis during stacking interactions through face $\mathrm{F}_{1}$.

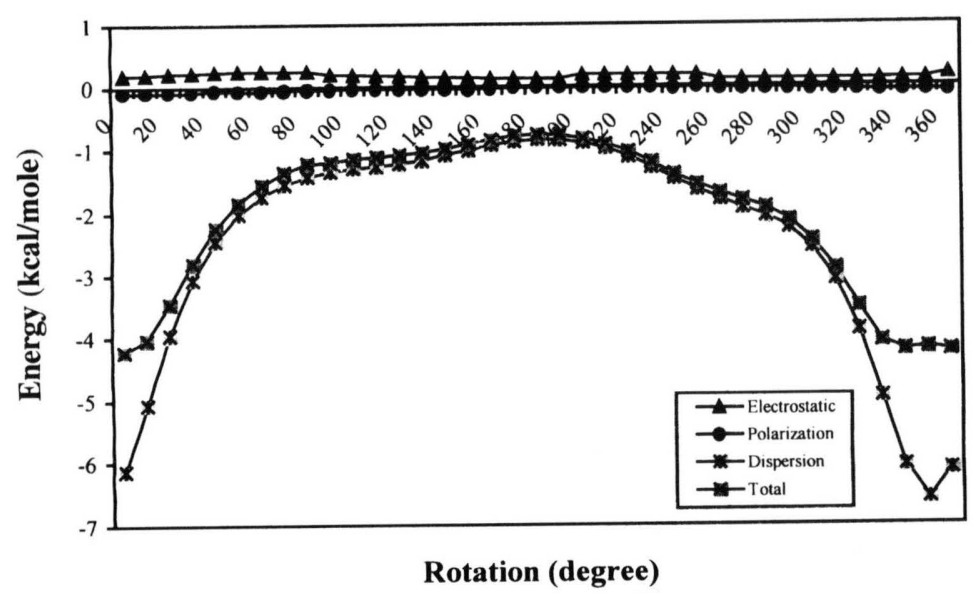

Fig. 4. Variation of the interaction energy components with respect to rotation about the $X$-axis during in-plane interactions through side $\mathrm{S}_{1}$.

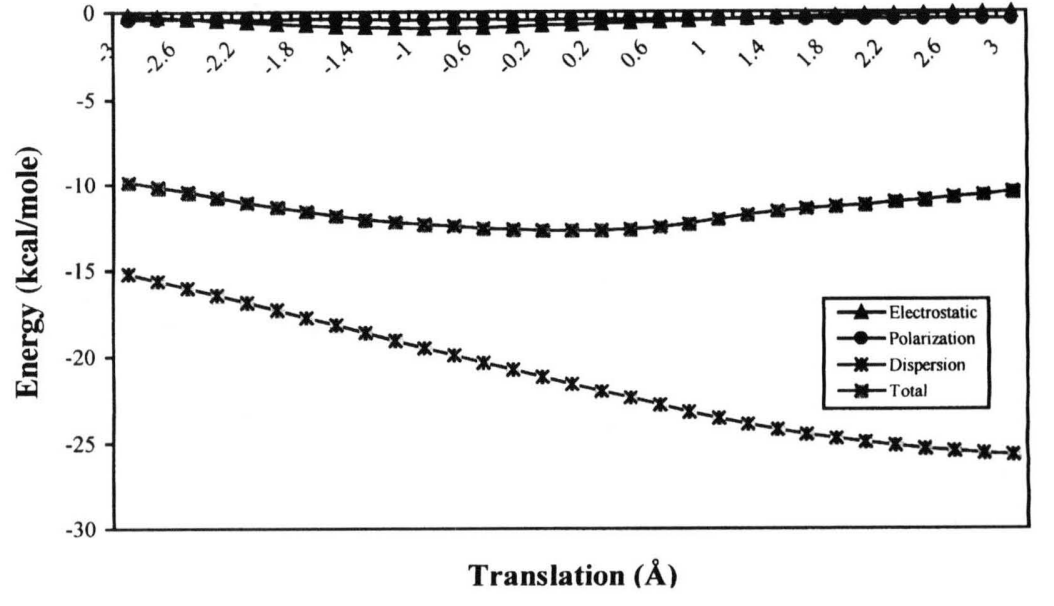

Fig. 3. Variation of the interaction energy components with respect to translation along the $X$-axis during stacking interactions through face $\mathrm{F}_{1}$.

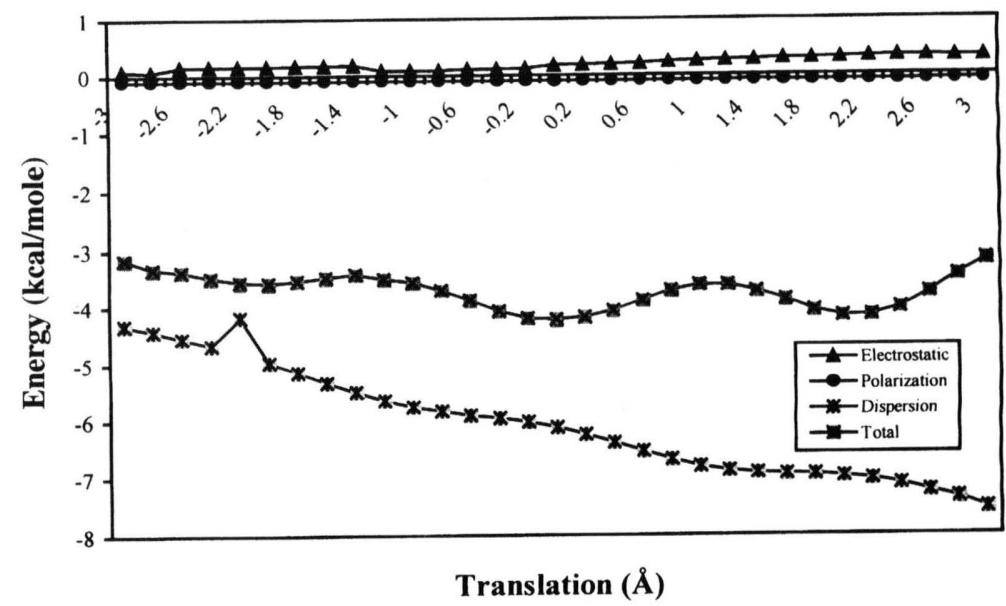

Fig. 5. Variation of the interaction energy components with respect to translation along the $X$-axis during in-plane interactions through side $\mathrm{S}_{1}$. 
although the optimum angle is always governed by Kitaigorodskii energies. For rotation in the range of $300^{\circ} \pm 10^{\circ}$, an energy difference of only $\sim 1 \mathrm{kcal} / \mathrm{mole}$ is observed, implying that on increased thermal agitation, the stacked molecule in a PBPCN pair may get oriented with respect to each other one. The contribution of polarization energy is negligible. The electrostatic energy term is much smaller than the dispersion term. The repulsion energy component has not been plotted, as it can easily be obtained with the help of dispersion and Kitaogorodskii curves.

The nematic character of liquid crystals is generally manifested by their translational freedom along the long molecular axis. Therefore, translations have been allowed at intervals of $0.2 \AA$, and the corresponding changes in the various interaction energies through face $F_{1}$ are reported in Figure 3. All components increase with increased overlapping, the increase being small for the electrostatic and polarization energies. The dispersion energy is mainly responsible for the attraction between the planes of the PBPCN molecules, though the exact optimum results always from the $\mathrm{Ki}$ taigorodskii energy curve, which has gross similarity with the total energy curve. Evidently, the stacked pair of PBPCN molecules can slide in the range of $2.6 \AA$ $\pm 0.4 \AA$ without any significant change in the energy and, hence, is capable retaining molecular order upto $3 \AA$ against increased thermal agitation.

\section{In-plane Interactions}

The interacting molecule has been kept at separation of $7 \AA$ along the $Y$-axis with respect to the fixed one to avoid van der Waals contacts. Similar calculations have been performed for in-plane interactions on the sides $\mathrm{S}_{1}$ and $\mathrm{S}_{2}$, i. e. at an intermediate distance +7 $\AA$ ) and $-7 \AA$, respectively, of the molecule. Again, rotations about the $Y$-and $X$-axis have been given and corresponding energies have been calculated. The energy has been minimized with respect to translation and rotation about all axes. The lowest in-plane geometry was thus obtained through side $S_{1}$ at a separation of $6.5 \AA$ with energy $-4.23 \mathrm{kcal} / \mathrm{mole}$ and side $\mathrm{S}_{2}$ at a separation of $6.3 \mathrm{~A}$ with energy $-5.01 \mathrm{kcal} / \mathrm{mole}$.

Figure 4 shows the variation of the interaction energy components through side $S_{1}$ with respect to rotation about the $X$-axis. The main attractive part of the energy comes through the dispersion term. It may be noted that side $S_{1}$ shows a clear preference, though the difference in energy for different values of rotation is
Table 2. Minimum energy values obtained between a pair of PBPCN molecules during stacking, in-plane and terminal interactions after refinement. Energy is expressed in $\mathrm{kcal} / \mathrm{mole}$.

\begin{tabular}{|c|c|c|c|c|c|c|}
\hline \multirow{2}{*}{$\begin{array}{l}\text { Energy } \\
\text { terms }\end{array}$} & \multicolumn{2}{|c|}{ Stacking Energy } & \multicolumn{2}{|c|}{ In-plane Energy } & \multicolumn{2}{|c|}{ Terminal Energy } \\
\hline & $+7 \AA$ & $-7 \AA$ & $+7 \AA$ & $-7 \AA$ & $+20 \AA$ & $-20 \AA$ \\
\hline & & 19 & & 0.2 & 0 & 0.019 \\
\hline$E_{\mathrm{OMI}}$ & 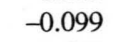 & -0.1 & 0 & 0.226 & $0 .($ & 0.001 \\
\hline$E_{\mathrm{MI}}^{\mathrm{VIN}}$ & -0.602 & -0.954 & 0.029 & 0.069 & -4.720 & 0.009 \\
\hline$E_{\mathrm{el}}$ & -0.725 & -0.925 & 0.180 & 0.531 & -3. & 0.031 \\
\hline$E$ & -0.431 & -0.695 & -0.110 & -0.099 & -0.208 & -0.011 \\
\hline$E_{\text {disp }}^{\text {por }}$ & -21.568 & -18.033 & -6.129 & -7.504 & -1.212 & -2.915 \\
\hline$E_{\text {rep }}$ & 9.991 & 6.819 & 1.822 & 2.065 & 2.026 & 0.899 \\
\hline$E_{\text {total }}$ & -12.729 & -12.834 & -4.228 & -5.009 & -3.166 & -1.996 \\
\hline
\end{tabular}

very small. Thus, generally, the molecule may be assumed to be capable of free rotations except at lower temperatures, where the two molecules prefer being in the same plane. The effect of translation along the $X$-axis is shown in Figure 5. It may be observed that the translational freedom is much more pronounced than the stacking interactions (Fig. 3).

\section{Terminal Interactions}

The length of molecule is approximately $17 \AA$, to investigate the terminal interactions away from the van der Waals contacts, the interacting molecule has been shifted along the $X$-axis by $\pm 20 \AA$ with respect to the fixed one and allowed to rotate along the $X$-and $Y$-axes. The energies at such points have been examined. Terminal interactions are much weaker than the stacking or in-plane interactions. Rotations about the $X$-axis show absolutely no preference for any angle, i.e. the molecules are free to rotate about their long molecular axis.

The interaction energy calculation can be correlated with the mesomorphic behaviour of the system. When solid crystals of PBPCN are heated, thermal vibrations disturb the molecular order of the strongly packed PBPCN molecules. Consequently, the attraction within a pair of molecules, largely comprising the dispersion forces, tend to get weaker at higher temperatures, and hence the possibility of relative movement within a molecular pair along the long molecular axis is considerably enhanced (Fig. 3). The freedom of molecules in a pair to slide along an axis perpendicular to the long molecular axis ( $Y$-axis) is energetically restricted, while terminal interactions are quite insignificant. 
The results favour the nematic behaviour of the system. At very high temperature, breaking of all dispersion forces results and all possible stacking geometries (even perpendicular stacking) become equally probable, which ultimately causes the system to become an isotropic melt.

The most prominent energy minima of the abovementioned interactions are refined, and values thus obtained are listed in Table 2 with all the contributing terms to enable comparison. These results indicate that the refinement corresponding to the stacking energy at face $F_{1}$ is maximum and the ultimate

[1] M. R. Wilson, in Hand Book of Liquid Crystals (D. Demus ed.), Wiley-VCH 1998.

[2] S. Chandrasekhar, Liquid Crystals, University Press, Cambridge 1992.

[3] P. G. de Gennes and J. Prost, The Physics of Liquid Crystals, Clarendon Press, Oxford 1993.

[4] N. K. Sanyal, S. N.Tiwari, and M. Roychoudhury, J. Phys.Soc. Japan 55, 1171 (1986).

[5] N. K. Sanyal, S. N.Tiwari, and M. Roychoudhury, Mol. Cryst. Liq. Cryst. 140, 179 (1986).

[6] M. Roychoudhury and D. P. Ojha, Mol. Cryst. Liq. Cryst. 213, 73 (1992). magnitude of stacking is larger than in-plane and terminal interactions. Further, all possible geometrical arrangements between a molecular pair during stacking, in-plane and terminal interactions have been considered.

\section{Acknowledgements}

The financial support rendered by the Council of Scientific \& Industrial Research (Grant No. 03 (0932)/01/EMR-II) and the Department of Science \& Technology (Grant No. SP/S2/M-45/94), New Delhi, India is gratefully acknowledged.

[7] S. N. Tiwari, N. K. Sanyal, and M. Roychoudhury, Mol. Cryst. Liq. Cryst. 204, 111 (1991).

[8] D. P. Ojha, Phase Transitions. 72, 211 (2000).

[9] D. P. Ojha, Z.Naturforsch. 56a, 319 (2001).

[10] P. Mandal and S. Paul, Mol. Cryst. Liq. Cryst. 131, 223 (1985).

[11] J. A. Pople and D. L. Beveridge, Approximate Molecular Orbital Theory, Mc-Graw Hill, New York 1970.

[12] P. Claverie, in Intermolecular Interactions: From Diatomic to Biopolymers (B.Pullman ed.), John Wiley \& Sons, New York 1978, p. 69.

[13] R. Rein, Adv. Quant. Chem. 7, 335 (1973). 